SCIENTIFIC REPORT

\title{
Contrast sensitivity improves after brimonidine therapy in primary open angle glaucoma: a case for neuroprotection
}

\author{
D W Evans, S L Hosking, D Gherghel, J D Bartlett
}

See end of article for

authors' affiliations

Br J Ophthalmol 2003;87:1463-1465

Correspondence to: Sarah L Hosking, Neuroscience Research Institute, Aston University, Aston Triangle, Birmingham B4 7ET, UK: s.l.hosking@aston.ac.uk

Accepted for publication 17 March 2003
Aims: To determine the visual outcome following initiation of brimonidine therapy in glaucoma. Methods: 16 newly diagnosed previously untreated glaucoma patients were randomly assigned to either timolol $0.5 \%$ or brimonidine $0.2 \%$. Visual acuity, contrast sensitivity (CS), visual fields, intraocular pressure (IOP), blood pressure, and heart rate were evaluated at baseline and after 3 months.

Results: IOP reduction was similar for both groups $(p<0.05)$. Brimonidine improved $C S$; in the right eye at 6 and $12 \mathrm{cpd}(p=0.043, p=0.017)$; in the left eye at 3 and $12 \mathrm{cpd}(p=0.044, p=0.046)$. Timolol reduced CS at $18 \mathrm{cpd}$ in the right eye $(p=0.041)$. There was no change in any other measured parameters.

Conclusion: Glaucoma patients exhibit improved CS on initiation of brimonidine therapy.

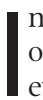
ncreased intraocular pressure (IOP) is the risk factor most often associated with glaucoma; however, there is strong evidence to suggest that there are pressure independent mechanisms associated with the development of the disease. ${ }^{1}$

The optic nerve damage in glaucoma affects contrast sensitivity to a greater extent than visual acuity. ${ }^{23}$ It has also been shown that contrast sensitivity improves on initiation of ocular hypotensive therapy, either through the use of $\beta$ blockers $^{4}$ or following surgical reduction of IOP. ${ }^{5}$ There is accumulating evidence that IOP lowering augmented with other therapeutic strategies limits further neurodegeneration. Drugs with neuroprotective properties are most likely to preserve or even improve visual function on initiation of therapy.

In this study we investigate the hypothesis that glaucoma therapy with brimonidine would provide greater benefit to central visual function than a drug such as timolol, which has similar IOP reducing characteristics, but no known neuroprotective properties.

\section{METHODS}

Sixteen newly diagnosed and previously untreated POAG patients were included in the study. Patients were diagnosed as having POAG if on two separate occasions they had IOP greater than $21 \mathrm{~mm} \mathrm{Hg}$, glaucomatous cupping of the optic disc, open anterior chamber angles, and glaucomatous visual field (VF) defects by Humphrey visual field analyser (ZeissHumphrey, San Leandro, CA, USA; program 24-2). Ocular exclusion criteria included visual acuity less than 20/30 (0.2 logMAR), IOP $>35 \mathrm{~mm} \mathrm{Hg}$, non-glaucomatous ocular disease, and media opacities or neurological disorders that could induce VF or contrast sensitivity (CS) defects. Patients were also excluded if they were known to suffer systemic disorders including diabetes mellitus, cardiovascular diseases, or had any recent history of systemic medication that could affect intraocular pressure. The study was conducted in accordance with the tenets of the Declaration of Helsinki and patients provided written informed consent before commencement.

At baseline, patients underwent VF, ETDRS acuity, Goldmann tonometry, BP, and HR measurements. Central CS at 3, 6, 12, and 18 cycles/degree was measured wearing best refraction, using the CSV-1000E Contrast Testing Instrument (VectorVision, Dayton, OH, USA), and using a standardised luminance level of $85 \mathrm{~cd} / \mathrm{m}^{2}$. To avoid learning effects, ${ }^{4}$ two practice trials were conducted before the first baseline measurement and one before each subsequent visit.

Patients were randomised into one of two treatment groups with (a) timolol gel $0.5 \%$, or (b) brimonidine $0.2 \%$. Patients and investigators were masked to the treatment condition. For each patient, two bottles of the drug were provided, one for the morning and one for the evening dosage. For the timolol treatment group, the evening dosage was a vehicle placebo. Patients returned after 3 months to be tested for IOP, CS, VF, BP, and HR.

The pre-treatment and post-treatment differences in IOP, CS, VF, BP, and HR for the two study groups were assessed

Table 1 Baseline characteristics for the study samples

\begin{tabular}{lccc}
\hline & Brimonidine (mean (SD)) & Timolol (mean (SD)) & p Value \\
\hline Age (years) & $57.86(8.59)$ & $60.11(10.91)$ & $>0.05$ \\
Visual acuity RE (logMAR) & $0.01(0.06)$ & $0.07(0.06)$ & $>0.05$ \\
Visual acuity LE (logMAR) & $0.003(0.07)$ & $0.09(0.08)$ & 0.0443 \\
IOP RE (mm Hg) & $22.31(6.10)$ & $24.31(5.69)$ & $>0.05$ \\
IOP LE (mm Hg) & $22.35(6.26)$ & $22.58(5.16)$ & $>0.05$ \\
MD RE (dB) & $-4.15(3.78)$ & $-6.80(4.48)$ & $>0.05$ \\
MD LE (dB) & $-5.82(4.04)$ & $-6.96(8.78)$ & $>0.05$ \\
Systolic BP (mm Hg) & $136.43(12.49)$ & $142.55(12.50)$ & $>0.05$ \\
Diastolic BP (mm Hg) & $86.00(7.39)$ & $84.89(8.13)$ & $>0.05$ \\
Mean BP (mm Hg) & $102.81(7.94)$ & $104.11(8.25)$ & $>0.05$ \\
Heart Rate (bpm) & $76.43(11.54)$ & $77.63(14.00)$ & $>0.05$ \\
\hline
\end{tabular}

$\mathrm{BP}=$ blood pressure, $\mathrm{IOP}=$ intraocular pressure, $M D=$ visual field mean defect, $\mathrm{RE}=$ right eye, $\mathrm{LE}=$ left eye. 

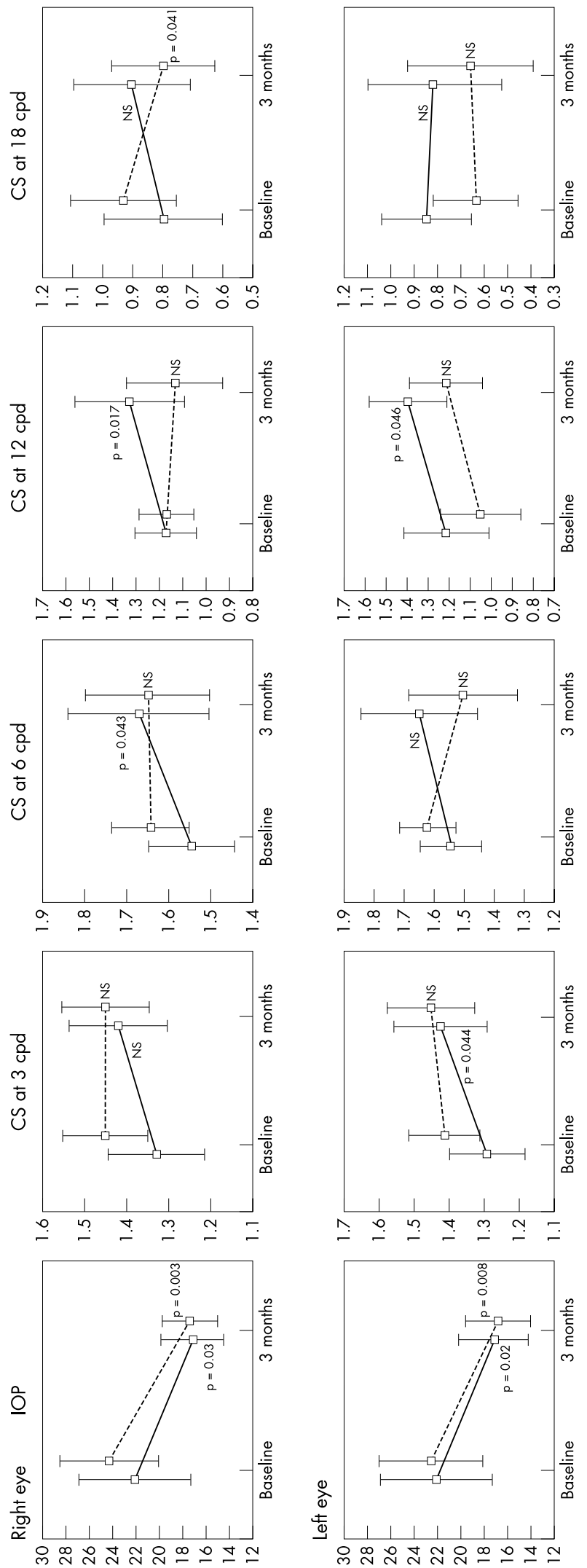

Figure 1 Graphs showing the baseline findings and 3 month posttreatment data for patients treated with brimonidine $0.2 \%$ (solid lines) and timolol $0.5 \%$ (broken lines) for the right eye (above) and left eye (below). Data shown (from left to right) include the IOP $(\mathrm{mm} \mathrm{Hg})$, and CS at $3,6,12$, and $18 \mathrm{cpd}$. Where significant change occurred the $p$ value is shown; NS = not significant. using repeated measures analysis of variance (REANOVA). A $\mathrm{p}$ value of less than 0.05 was considered statistically significant.

\section{RESULTS}

At baseline, the groups were similar for age, IOP, VF mean deviation, systemic BP, and HR; visual acuity was slightly worse in the left eye of the timolol treated group $(\mathrm{p}=0.044)$ (Table 1).

The IOP reduction after 3 months of treatment was statistically significant and similar in both study groups (Fig 1).

After 3 months of treatment there was a significant improvement in contrast sensitivity in the brimonidine treated group; for 6 and 12 cycles/degree in the right eye $(\mathrm{p}=0.043 ; \mathrm{p}=0.017$ respectively), and for the 3 and 12 cycles/degree in the left eye $(p=0.044 ; p=0.046$ respectively). In contrast, the timolol treated group demonstrated a significant decrease in contrast sensitivity in the $\mathrm{RE}$ at 18 cycles/degree $(p=0.041)$ and no other change. These effects are summarised in Figure 1. There was no change in $\mathrm{VF}, \mathrm{BP}$, or HR after 3 months of treatment $(\mathrm{p}>0.05)$.

\section{DISCUSSION}

In this study, contrast sensitivity improved after 3 months of treatment with brimonidine $0.2 \%$, while timolol therapy resulted in no improvement but some deterioration in CS. The IOP lowering effects of the two drugs were similar, suggesting an IOP independent mechanism for the visual outcome.

During a disease process, subpopulations of cells may be dead, dying, or healthy depending on the degree and duration of the disease. It is likely that improvements in function such as those reported here, and previously, ${ }^{45}$ could be the result of recovery of function in dying cells that return to a healthier status following treatment. This is not to suggest that improvements are progressive, but most likely a stepped response to the onset of therapy.

The density of ganglion cells is around 70 -fold greater in the central 3 degrees than that at 20 degrees eccentricity where a nasal step VF defect typically appears. ${ }^{6}$ This high density of retinal ganglion cells in central vision may result in oversampling or visual redundancy, during most visual function investigations and a relatively late detection of defects in the central visual field. Contrast sensitivity is a more sensitive test enabling subtle defects or improvements to be detected centrally in the early stages of disease. Any visual improvement in the neurological functioning of the retinal ganglion cells would be demonstrated only where test variability was extremely low, such as in central vision. Investigations of visual function that combine low variability and a low sampling density are most likely to demonstrate any subtle functional improvements, as shown in this study.

Brimonidine has a known neuroprotective effect, probably linked to the alpha-2 receptor and its signalling pathway. ${ }^{7-9}$ Alpha-2 receptors are present in the retina, ${ }^{10}$ and alpha-2 agonists such as brimonidine have exhibited neuroprotective properties following crush injury, ${ }^{11}$ pressure induced retinal ischaemia ${ }^{12}$ and chronic elevation of intraocular pressure. ${ }^{13}$ Recently, WoldeMussie, et a l $^{14}$ demonstrated a dose dependent reduction in progression of retinal ganglion cell loss following administration of brimonidine which was not present using timolol, and not linked to IOP lowering effects.

Treatment related perfusion changes unrelated to IOP affect contrast sensitivity. ${ }^{15} 16$ Blood velocity in the ophthalmic artery is highly correlated with changes in contrast sensitivity in normal tension glaucoma patients following treatment with nifedipine. ${ }^{17}$ However, no such association has been found in POAG patients treated with either timolol 
or brimonidine ${ }^{15}$ and an alternative, possibly neuroprotective, mechanism for the improvement in contrast sensitivity following treatment with brimonidine should be considered.

These data clearly demonstrate that CS improves on initiation of brimonidine therapy in glaucoma. This finding does not appear to be related to IOP lowering effects but rather may be linked to the neuroprotective capabilities of this therapy.

\section{Authors' affiliations}

D W Evans, School of Optometry, University of Alabama at Birmingham, Birmingham, AL, USA

S L Hosking, D Gherghel, Neuroscience Research Institute, Aston University, Aston Triangle, Birmingham B4 7ET, UK

J D Bartlett, School of Optometry, University of Alabama at Birmingham, and Department of Pharmacology and Toxicology, School of Medicine, University of Alabama, Birmingham, AL, USA

\section{REFERENCES}

1 Flammer J, Orgül S. Optic nerve blood-flow abnormalities in glaucoma. Prog Ret Eye Res 1998;17:267-89.

2 Stamper RL. Psychophysical changes in glaucoma. Surv Ophthalmol 1989;33(suppl I):309-18.

3 Sample PA, Juang PS, Weinreb RN. Isolating the effects of primary openangle glaucoma on the contrast sensitivity function. Am J Ophthalmol $1991 ; 112: 308-16$.

4 Pomerance GN, Evans DW. Test-retest reliability of the CSV-1000 contrast test and its relationship to glaucoma therapy. Invest Ophthalmol Vis Sci 1994;35:3357-61.
5 Gandolfi SA, Cionino L, Vecchi M. Improvement of spatial contrast sensitivity threshold after surgical reduction of intraocular pressure in unilateral hightension glaucoma. Acta Ophthalmol Scand Suppl 1997;224:40.

6 Curcio C, Allen K. Topography of ganglion cells in human retina. J Comp Neurol 1990;300:5-25.

7 Donello JE, Padillo EU, Webster ML, et al. Alpha(2)-adrenoreceptor agonists inhibit vitreal glutamate and aspartate accumulation and preserve retinal function after transient ischemia. J Pharmacol Exp Ther 2001;296:216-23.

8 Hasson D, Lai R, Chun T, et al. Functional protection of rat retina from ischemic injury by brimonidine. Soc Neurosci Abstracts 1997;23:168.

9 Lai R, Chun T, Hasson D, et al. Activation of cell survival signaling pathway in the retina by selective alpha-2 adrenoreceptor agonist brimonidine. Invest Ophthalmol Vis Sci 1999;40(Suppl):S763.

10 Bylund DB, Chacko DM. Characterization of alpha2 adrenergic receptor subtypes in human ocular tissue homogenates. Invest Ophthalmol Vis Sci 1999;40:2299-306.

11 Yoles E, Wheeler L, Schwartz M. Alpha2-adrenoreceptor agonists are neuroprotective in a rat model of optic nerve degeneration. Invest Ophthalmol Vis Sci 1999:40:65-73

12 Wheeler LA, Lai R, Wolde-Mussie E. From the lab to the clinic: Activation of an alpha2 agonist pathway is neuroprotective in models of retinal and optic nerve injury. Eur J Ophthalmol 1999;9:S17-21.

13 Wolde-Mussie E, Ruiz G, Wijono M, et al. Neuroprotective effect of brimonidine in chronic ocular hypertensive rats. Invest Ophthalmol Vis Sci 2000;41:\$830

14 Wolde-Mussie E, Ruiz G, Wijono M, et al. Neuroprotection of retinal ganglion cells by brimonidine in rats with laser-induced chronic ocula hypertension. Invest Ophthalmol Vis Sci 2001;42:2849-55.

15 Evans DW, Harris A, Chung HS, et al. Effects of long-term hypotensive therapy with nonselective beta-blockers on ocular hemodynamics in primary open-angle glaucoma. J Glaucoma 1999;8:12-17.

16 Evans D, Harris A, Cantor LB. Primary open-angle glaucoma patients characterized by ocular vasospasm demonstrate a different ocular vascular response to timolol versus betaxolol. J Ocul Pharmacol Ther 1999; 15:479-87.

17 Harris A, Evans DW, Cantor LB, et al. Hemodynamic and visual function effects of oral nifedipine in. Am J Ophthalmol 1997;124:296-302. 